TRANSACTIONS OF THE

AMERICAN MATHEMATICAL SOCIETY

Volume 363, Number 1, January 2011, Pages 331-343

S 0002-9947(2010)05049-4

Article electronically published on August 25, 2010

\title{
EXTENDING LOCAL ANALYTIC CONJUGACIES
}

\author{
HIROYUKI INOU
}

\begin{abstract}
We prove that if two globally-defined one-dimensional complex dynamics are locally analytically conjugate, then we extend the conjugacy to obtain global conjugacy by a correspondence. The most important case occurs when two rational maps have analytically conjugate polynomial-like restrictions. In this case, we prove that there exists another rational map which is semiconjugate to them both by some rational maps.
\end{abstract}

\section{INTRODUCTION}

Polynomial-like mappings were introduced by Douady and Hubbard [DH. The definition of a polynomial-like mapping is very simple and it contains large classes of maps: A map $f: U^{\prime} \rightarrow U$ is polynomial-like if it is proper and holomorphic, $U^{\prime}$ and $U$ are topological disks in $\mathbb{C}$, and $U^{\prime}$ is a relatively compact subset of $U$. However, they proved that polynomial-like mappings actually behave like polynomials. Namely, any polynomial-like map is hybrid equivalent to some polynomial of the same degree (the straightening theorem). In other words, the set of hybrid equivalence classes of polynomial-like mappings of a given degree $d$ is very small; it is just the hybrid equivalence classes of polynomials of degree $d$.

However, in this paper, we prove there are plenty of classes in the sense of analytic equivalence, by showing that we can distinguish polynomial-like renormalizations (or restrictions) of rational maps by their analytic equivalence classes except when they have some global analytic correspondence. We use this theorem to show that straightening maps between renormalizable polynomials of a given combinatorics and the corresponding family of polynomials is always discontinuous [I] .

Theorem 1. For $i=1,2$, let $f_{i}$ be a rational map or an entire map. Assume that there exist polynomial-like restrictions $f_{1}: U_{1}^{\prime} \rightarrow U_{1}$ and $f_{2}: U_{2}^{\prime} \rightarrow U_{2}$ of degree not less than two which are analytically conjugate. Then there exist rational or entire maps $g, \phi_{1}$ and $\phi_{2}$ such that

$$
f_{1} \circ \phi_{1}=\phi_{1} \circ g, \quad f_{2} \circ \phi_{2}=\phi_{2} \circ g
$$

and $g$ has a polynomial-like restriction $g: V^{\prime} \rightarrow V$ analytically conjugate to $f_{1}$ : $U_{1}^{\prime} \rightarrow U_{1}$ by $\phi_{1}$.

Furthermore,

- if both of the degrees $d_{1}=\operatorname{deg} f_{1}$ and $d_{2}=\operatorname{deg} f_{2}$ are finite, then $g, \phi_{1}, \phi_{2}$ are also of finite degrees. In particular, we have $d_{1}=d_{2}$.

Received by the editors September 12, 2008 and, in revised form, February 26, 2009

2010 Mathematics Subject Classification. Primary 37F10; Secondary 32B10, 30D05.

(C)2010 American Mathematical Society 
- If $f_{1}$ is a polynomial and $f_{2}$ is a rational map, then $f_{2}$ is a polynomial by taking a Möbius conjugate and we can take $g, \phi_{1}$ and $\phi_{2}$ to be polynomials.

Here we give several examples such that the conclusion holds. In the following, we say a map $g$ is semiconjugate to another map $f$ if there exists a map $h$ such that $h \circ g=f \circ h$. We call such an $h$ a semiconjugacy from $g$ to $f$. In this paper, we always assume that $f, g$ and $h$ are holomorphic and mainly we are interested in global semiconjugacies, i.e., $f, g$ and $h$ are polynomials, rational maps, or entire maps.

Example. (1) Let $h(z)$ be a polynomial and let $f(z)=z(h(z))^{d}, g(z)=z h\left(z^{d}\right)$ and $\phi(z)=z^{d}$. Then $\phi(g(z))=f(\phi(z))$.

(2) For rational maps $R_{1}$ and $R_{2}$, let $f=R_{1} \circ R_{2}$ and $g=R_{2} \circ R_{1}$. Then $f$ and $g$ are semiconjugate to each other by $R_{1}$ and $R_{2}$.

(3) Similarly, let $R_{1}, R_{2}$ and $R_{3}$ be rational maps and let $f_{1}=R_{1} \circ R_{2} \circ R_{3}$, $f_{2}=R_{2} \circ R_{3} \circ R_{1}$ and $g=R_{3} \circ R_{1} \circ R_{2}$. Then $g$ is semiconjugate to $f_{i}$ by $\phi_{i}$, where $\phi_{1}=R_{3}$ and $\phi_{2}=R_{3} \circ R_{1}$.

(4) Let $f_{1}(z)=z\left(c+z^{2}\right)^{3}, f_{2}(z)=z\left(c+z^{3}\right)^{2}$ and $g(z)=z\left(c+z^{6}\right)$. Then $f_{1}\left(z^{3}\right)=(g(z))^{3}$ and $\left.f_{2}\left(z^{2}\right)=(g(z))\right)^{2}$. Furthermore, $f_{1}$ and $f_{2}$ are not semiconjugate to each other except when $c=0$ (see Appendix $\mathrm{A}$ ).

Ritt $[\mathrm{R}$ gave a description of decompositions of polynomials by composition. It gives a classification of semiconjugate polynomials. See Appendix A for more details. A classification of semiconjugate rational maps is an open problem because we do not have such a nice theory of decompositions of rational maps. Transcendental entire maps seem to be more difficult. We do not even know whether there exist a transcendental entire map $f_{1}$ and a rational map $f_{2}$ which satisfy the conclusion of the theorem. A transcendental entire map cannot be semiconjugate to a polynomial by a (transcendental) entire map (Proposition 4), but that is almost all that we know and the following are open:

Conjecture. (1) A polynomial of degree greater than one cannot be semiconjugate to a transcendental entire map by an entire map.

(2) A transcendental entire map cannot be semiconjugate to a rational map by a transcendental meromorphic map defined on $\mathbb{C}$.

Observe that the inverse of a linearizing coordinate of a repelling fixed point gives a semiconjugacy from a linear map (polynomial of degree one) to a transcendental entire map.

The conclusion (11) in the theorem can be considered as a relation between $f_{1}$ and $f_{2}$. It becomes clear by describing it in terms of correspondences [BS. A holomorphic correspondence $h: X \rightarrow Y$ is a multi-valued map between Riemann surfaces which has the form $h=\tilde{Q}_{+} \circ \tilde{Q}_{-}^{-1}$, where $\tilde{Q}_{-}: Z \rightarrow X$ and $\tilde{Q}_{+}: Z \rightarrow Y$ are holomorphic maps between complex manifolds. That is, $z \mapsto w$ if there exists some $x \in Z$ such that $z=\tilde{Q}_{-}(x)$ and $w=\tilde{Q}_{+}(x)$. If $X$ and $Y$ are compact, then the graph $\Gamma=\left\{\left(\tilde{Q}_{-}(x), \tilde{Q}_{+}(x)\right) ; x \in Z\right\}$ is a (singular) one-dimensional complex manifold. Conversely, any (singular) one-dimensional complex manifold defines a holomorphic correspondence.

In our case, $h=\phi_{2} \circ \phi_{1}^{-1}$ is a correspondence conjugating $f_{1}$ and $f_{2}$. Furthermore, if $f_{1}$ and $f_{2}$ are rational, then the graph of $h$ is an irreducible algebraic set in $\hat{\mathbb{C}}^{2}$ (see Proposition (3). Hence we can say that $f_{1}$ and $f_{2}$ are conjugate by 
an irreducible holomorphic correspondence. In the case of (transcendental) entire maps, the graph can have a very wild shape, but we still require $Z$ to be a Riemann surface (i.e. connected one-dimensional complex manifold). It is an open problem whether this relation is an equivalence relation or not. We can compose conjugacies as correspondences to get a conjugacy, but a composition of two irreducible holomorphic correspondences is not irreducible in general.

The proof of Theorem 1 depends on the "graph pushing-forward argument", which is a generalization of the classical extension technique by a functional equation. If we have a local conjugacy and one of the dynamics is univalent (like a linearization of a fixed point), we can extend the conjugacy from univalent dynamics to the other globally by using a functional equation.

Even when both dynamics have critical points, we can still extend a local conjugacy by pushing forward the conjugacy as a correspondence. The precise statement is given in Theorem 2 ,

Roughly speaking, consider the graph $\Gamma_{0} \subset U_{1} \times U_{2}$ of the analytic conjugacy $\phi$ and the product dynamics $F=\left(f_{1}, f_{2}\right)$. Then $\Gamma_{n}:=F^{n}\left(\Gamma_{0}\right)$ forms an increasing sequence of local analytic sets. The union $\Gamma=\bigcup_{n} \Gamma_{n}$ is an invariant local analytic set, so we have a dynamics $F: \Gamma \rightarrow \Gamma$. By normalizing (desingularizing) $\Gamma$, we obtain a Riemann surface $X$ and a dynamics $g: X \rightarrow X$ on it. We also naturally have semiconjugacies $\phi_{i}: X \rightarrow \hat{\mathbb{C}}$ from $g$ to $f_{i}$. Since $g$ has a chaotic dynamics coming from the original polynomial-like maps, $X$ cannot be a hyperbolic Riemann surface. Hence we can classify $X$ and study the dynamics $g$ to obtain the theorem. Note that this proves that $\Gamma$ is not only analytic but also algebraic in fact (Proposition 3). Furthermore, $X$ is minimal in the sense that if there exist rational maps $\hat{g}$ and $\hat{\phi}_{i}$ such that $\hat{\phi}_{i} \circ \hat{g}=f_{i} \circ \hat{\phi}_{i}$, then $\hat{g}$ is semiconjugate to $g$ (Proposition 6).

If we allow polynomial-like restrictions $f_{i}: U_{i}^{\prime} \rightarrow U_{i}$ to have degree one, then they are simply neighborhoods of repelling fixed points and they are analytically conjugate if and only if those fixed points have the same multiplier. Our construction still works well in that case, but usually we have that $g: \mathbb{C} \rightarrow \mathbb{C}$ is a linear map and $\phi_{1}: \mathbb{C} \rightarrow \hat{\mathbb{C}}$ and $\phi_{2}: \mathbb{C} \rightarrow \hat{\mathbb{C}}$ are the inverses of linearizing coordinates for those fixed points. Thus $\phi_{1}$ and $\phi_{2}$ are transcendental. Further when $f_{1}$ and $f_{2}$ are rational maps, we have the following classification for a triple $\left(f_{i}, g, \phi_{i}\right)$ for each $i=1,2$ :

(1) The conclusion of Theorem 1 as in the above examples.

(2) Power maps: $X=\mathbb{C}^{*}$ and $g(z)=z^{ \pm d}$. If further we assume that $\phi_{i}$ is not a rational map, then $f_{i}$ is an integral Lattès example.

(3) Chebyshev maps: $X=\mathbb{C}$ and $g\left(z+z^{-1}\right)=z^{d}+z^{-d}$. Similar to the case of power maps, if $\phi_{i}$ is not rational, $f_{i}$ is an integral Lattès example.

(4) Linear maps on tori: $X=\mathbb{C} / \Lambda$ is a torus and $g([z])=[\lambda z]$ for some $\lambda$. Then $f_{i}$ is a Lattès example.

In particular, $g$ is not transcendental even in this case. Note that the definition of the Chebyshev polynomial is slightly different from the usual definition (use $\left(z+z^{-1}\right) / 2$ instead of $\left.z+z^{-1}\right)$, but they are linearly conjugate and we use the above definition to simplify the notation. A Lattès example is a rational map $f: \hat{\mathbb{C}} \rightarrow \hat{\mathbb{C}}$ such that there exists a doubly periodic meromorphic function $\theta: \mathbb{C} \rightarrow \hat{\mathbb{C}}$ and an affine map $\ell(t)=a t+b$ such that $\ell \circ \theta=f \circ \ell$. We say $f$ is integral if $a \in \mathbb{Z}$. For more details, see $[\mathrm{M}]$ and $[\mathrm{BE}]$. 


\section{Constructing a COMPlex manifold}

Let $R_{1}$ and $R_{2}$ be Riemann surfaces and let $M=R_{1} \times R_{2}$. In the following, we denote by $p_{i}: M \rightarrow R_{i}$ the projection to the $i$-th coordinate for $i=1,2$.

In this section, we prove the following desingularization theorem:

Theorem 2. Let $R_{1}$ and $R_{2}$ be Riemann surfaces and let $M=R_{1} \times R_{2}$. Let $f_{i}: R_{i} \rightarrow R_{i}$ be a holomorphic self-map on $R_{i}$ for $i=1,2, \phi: U_{1} \rightarrow U_{2}$ be a nonconstant holomorphic map between open sets $U_{i} \subset R_{i}$ and $\Gamma_{0}=\left\{\left(z, \phi_{z}\right) ; z \in\right.$ $\left.U_{1}\right\} \subset M$ be the graph of $\phi$. Consider a forward invariant set

$$
\Gamma=\bigcup_{n \geq 0} F^{n}\left(\Gamma_{0}\right)
$$

by the product map $F\left(z_{1}, z_{2}\right)=\left(f_{1}\left(z_{1}\right), f_{2}\left(z_{2}\right)\right)$ on $M$. Then there exist a onedimensional complex manifold $X$, possibly having infinitely many connected components, and holomorphic maps $g: X \rightarrow X$ and $\pi: X \rightarrow M$ such that $\pi(X)=\Gamma$ and $\pi \circ g=F \circ \pi$. Furthermore,

(1) let us denote $\phi_{i}=p_{i} \circ \pi$. There exists an open set $U \subset X$ such that $\pi(U)=\Gamma_{0}$ and $\phi_{i}: U \rightarrow U_{1}$ is an isomorphism.

(2) If the degrees of $f_{1}$ and $f_{2}$ are finite, then $\operatorname{deg} g$ is not greater than $\operatorname{deg} f_{1}$. $\operatorname{deg} f_{2}$.

(3) If $U_{1}$ is connected and $\phi \circ f_{1}=f_{2} \circ \phi$ on some open set $U_{1}^{\prime} \subset U_{1}$ with $f_{1}\left(U_{1}^{\prime}\right) \subset U_{1}$, then $X$ is a Riemann surface (i.e., it is connected) and there exists an open set $U^{\prime} \subset U$ such that $\phi_{1}\left(U^{\prime}\right)=U, g\left(U^{\prime}\right) \subset U$ and $\phi_{1} \circ g=$ $f_{1} \circ \phi_{1}$ on $U^{\prime}$.

Note that $\Gamma$ can be weird, especially when $f_{i}$ is transcendental. It might have a lot of self-intersections and might accumulate to itself like (un)stable manifolds of two-dimensional dynamics, and so on. This theorem implies that we can still "desingularize" $\Gamma$.

Proof. First, observe that $\Gamma_{n}$ is a one-dimensional local analytic set in $M$ for each $n$. In fact, it is trivial for the graph $\Gamma_{0}$ of $\phi$, and $\Gamma_{n}$ is so because it is the image of a local analytic set by a proper map.

Let $\tilde{\Gamma}_{k}=\bigcup_{k=0}^{n} \Gamma_{k}$ and let $\pi_{n}: X_{n} \rightarrow \tilde{\Gamma}_{k}$ be the normalization (desingularization) of $\tilde{\Gamma}_{n}$; i.e., $X_{n}$ is a one-dimensional complex manifold and $\pi_{n}: X_{n} \rightarrow M$ is a finite proper holomorphic map which is biholomorphic to its image except on a discrete set (see, e.g., [C, §6]). We have a natural inclusion $\iota_{n}: X_{n} \rightarrow X_{n+1}$ induced from the inclusion $\tilde{\Gamma}_{k} \subset \tilde{\Gamma}_{k}$, which is holomorphic by the removable singularity theorem. Similarly, since we have $F\left(\tilde{\Gamma}_{k}\right) \subset \tilde{\Gamma}_{k+1}$, there exists a holomorphic map $g_{n}: X_{n} \rightarrow X_{n+1}$ such that $\pi_{n+1} \circ g_{n}=F \circ \pi_{n}$. We can take the direct limit of $\left\{X_{n}\right\}$ :

$$
X=\lim _{\longrightarrow} X_{n}
$$

Then $X$ is a complex manifold and $\pi_{n}$ and $g_{n}$ induce holomorphic maps $\pi: X \rightarrow$ $\Gamma \subset M$ (the normalization of $\Gamma$ ) and $g: X \rightarrow X$ such that $\pi \circ g=F \circ \pi$.

Let $U=\left\{[x] \in X ; x \in X_{0}\right\}$. It is easy to see that $U, X_{0}, \Gamma_{0}$ and $U_{i}$ are naturally isomorphic. This proves (11). The property (2) follows from the facts that $\pi$ is one-to-one except on a discrete set and $\operatorname{deg} F=\operatorname{deg} f_{1} \cdot \operatorname{deg} f_{2}$. 
Under the assumption in (3), we have $\Gamma_{n+1} \cap \Gamma_{n} \supset F^{n+1}\left(\Phi\left(U_{1}^{\prime}\right)\right)$, where $\Phi(z)=$ $(z, \phi(z))$ for $z \in U_{1}$. Therefore, $\tilde{\Gamma}_{n}$ is an irreducible local analytic set and it follows that $X_{n}$ is connected. Since $\phi_{1}: U \rightarrow U_{1}$ is an isomorphism by (11), it is easy to check that $U^{\prime}=\left(\left.\phi_{1}\right|_{U}\right)^{-1}\left(U_{1}^{\prime}\right)$ satisfies the desired property.

Although we use the removable singularity theorem to prove the analyticity of $g$, we can check directly the analyticity of the map $g$ (or $g_{n}$ ) at a singular point. For a point $x_{0} \in X_{n}$, there is a neighborhood $V \subset X_{n}$ of $x_{0}$ such that $\pi_{n}$ is biholomorphic on $V \backslash x_{0}$. Take a point $z_{0} \in U_{1}$ and $0 \leq k \leq n$ such that there exists a neighborhood $V_{1}$ of $z_{0}$ such that $F^{k}\left(\Phi\left(z_{0}\right)\right)=\pi_{n}\left(x_{0}\right)$ and

$$
F^{k} \circ \Phi:\left(V_{1} \backslash z_{0}\right) \rightarrow F^{k} \circ \Phi\left(V_{1}\right) \subset \pi_{n}\left(V \backslash x_{0}\right)
$$

is a proper map. Let $a_{1}$ be the local degree of $f_{1}^{k}$ at $z_{0}$. Then there exist conformal maps $\psi_{0}$ and $\psi_{1}$ defined near $z_{0}$ and $f_{1}^{k}\left(z_{0}\right)$, respectively, such that $\psi_{0}\left(z_{0}\right)=\psi_{1}\left(f_{1}^{k}\left(z_{0}\right)\right)=0$ and

$$
\psi_{1} \circ f_{1}^{k} \circ \psi_{0}^{-1}(w)=w^{a_{1}} .
$$

Therefore, by the equality $F^{k} \circ \Phi(z)=\left(f_{1}^{k}(z), f_{2}^{k}(\phi(z))\right)$, there exists some $b_{1}>0$ dividing $a_{1}$ such that $F^{k} \circ \Phi\left(\psi_{0}^{-1}(w)\right)=F^{k} \circ \Phi\left(\psi_{0}^{-1}(\tilde{w})\right)$ if and only if $w^{b_{1}}=\tilde{w}^{b_{1}}$. Thus it follows that $w^{b_{1}}$ gives a local coordinate (complex structure) of $X$ in a neighborhood of $x_{0}$.

Similarly, there exists a conformal map $\psi_{2}$ at $f_{1}^{k+1}\left(z_{0}\right)$ such that $\psi_{2}\left(f_{1}^{k+1}\left(z_{0}\right)\right)=$ 0 and $\psi_{2} \circ f_{1}^{k+1} \circ \psi_{0}^{-1}(w)=w^{a_{2}}$ where $a_{2}=\operatorname{deg}_{z_{0}}\left(f_{1}^{k+1}\right)$ by replacing $\psi_{0}$ if necessary and $w^{b_{2}}$ gives a local coordinate in a neighborhood of $g\left(x_{0}\right)$ for some $b_{2}$ which divides $a_{2}$. Thus $w^{b_{1}} \mapsto w^{b_{2}}$ is holomorphic because $b_{1}$ divides $b_{2}$. This proves that $g$ is holomorphic at $x_{0}$.

\section{Proof of The TheOREM}

3.1. Basic classification. Now assume that $f_{1}$ and $f_{2}$ are rational maps or entire maps, and $f_{1}: U_{1}^{\prime} \rightarrow U_{1}$ and $f_{2}: U_{2}^{\prime} \rightarrow U_{2}$ are polynomial-like mappings of degree $d^{\prime} \geq 2$ with analytic conjugacy $\phi: U_{1} \rightarrow U_{2}$. By Theorem 2, there exists a Riemann surface $X$ and a holomorphic map $g: X \rightarrow X$ such that $\phi_{i} \circ g=f_{i} \circ \tilde{\pi}_{i}$.

Furthermore, $U$ in the conclusion of Theorem 2 is naturally isomorphic to $U_{i}$ under $\phi_{i}$. Namely, $g$ has a polynomial-like restriction analytically conjugate to $f_{i}: U_{i}^{\prime} \rightarrow U_{i}$. This implies that $g$ has a chaotic dynamics (for example, $g$ has a repelling periodic point). Hence $X$ cannot be hyperbolic. Therefore, $X$ is conformally isomorphic to either the Riemann sphere $\hat{\mathbb{C}}$, the complex plane $\mathbb{C}$, the punctured plane $\mathbb{C}^{*}=\mathbb{C} \backslash\{0\}$, or a torus $\mathbb{C} / \Lambda$. Therefore, $g$ is

(1) a rational map if $X \cong \hat{\mathbb{C}}$,

(2) an entire map if $X \cong \mathbb{C}$,

(3) $g(z) \cong z^{ \pm \operatorname{deg} g} e^{h(z)}$ for some holomorphic map $h: \mathbb{C}^{*} \rightarrow \mathbb{C}$ if $X \cong \mathbb{C}^{*}$, and

(4) a linear map if $X$ is a torus.

However, if $X$ is a torus, $g$ cannot have a polynomial-like restriction of degree $d^{\prime} \geq 2$, so it is not the case.

Since $U_{i}$ is an open set intersecting the Julia set of $f_{i}$, we have

$$
\phi_{i}(X)=\bigcup_{n} f_{i}^{n}\left(U_{i}\right) \supset \hat{\mathbb{C}} \backslash \mathcal{E}_{i},
$$


where $\mathcal{E}_{i}$ is the exceptional set for $f_{i}$. Hence we may assume either

(1) $\phi_{i}(X)=\hat{\mathbb{C}}$,

(2) $\phi_{i}(X)=\mathbb{C}$ and $f_{i}$ is an entire map, or

(3) $\phi_{i}(X)=\mathbb{C}^{*}$.

Hence for each $i$, we can divide into cases according to $X$ and $\phi_{i}(X)$.

3.2. The case of rational maps. First, we consider the case of rational maps (including polynomials), i.e., $d_{i}=\operatorname{deg} f_{i}<\infty$. Then $d=\operatorname{deg} g$ is also finite by Theorem 2. Hence if $X \cong \mathbb{C}^{*}$, then $g(z)=z^{ \pm d}$ does not have a polynomial-like restriction in $\mathbb{C}^{*}$. Hence we may assume $X=\hat{\mathbb{C}}$ or $\mathbb{C}$.

Similarly, nor is $\phi_{i}(X)=\mathbb{C}^{*}$ the case. Thus we need only consider the cases $\phi_{i}(X)=\hat{\mathbb{C}}$ and $\phi_{i}(X)=\mathbb{C}$.

Case I. $\phi_{i}(X)=\hat{\mathbb{C}}$ and $X=\hat{\mathbb{C}}$. Then clearly $\phi_{i}: \hat{\mathbb{C}} \rightarrow \hat{\mathbb{C}}$ is a rational map and we are done.

Case II. $\phi_{i}(X)=\hat{\mathbb{C}}$ and $X=\mathbb{C}$. A priori, $\phi_{i}$ can be transcendental. However, Eremenko [Er] proved that $\phi_{i}$ is transcendental if $g$ is either a power map or a Chebyshev map, and $f_{i}$ is an integral Lattès example. Since any integral Lattès example does not have a polynomial-like restriction, this cannot happen. Thus $\phi_{i}$ is a polynomial. In particular, we have $\phi_{i}(X)=\mathbb{C}$, so Case II does not occur indeed.

Case III. $\phi_{i}(X)=\mathbb{C}$ and $X=\hat{\mathbb{C}}$. Then $\phi_{i}$ must be a constant, which is a contradiction. Therefore, we do not have this case either.

Case IV. $\phi_{i}(X)=\mathbb{C}$ and $X=\mathbb{C}$. Again by $\left[\mathrm{Er}, \phi_{i}\right.$ cannot be a transcendental entire function, so $\phi_{i}$ is a polynomial.

In any case, $\phi_{i}$ is a rational map. Moreover, if $f_{1}$ is a polynomial, then we have $\phi_{1}(X)=\mathbb{C}$ by construction. This implies that $X=\mathbb{C}$ and $\phi_{2}(X)=\mathbb{C}$. This proves Theorem 1 for rational maps.

Proposition 3. Under the assumption of Theorem 1, if $f_{1}$ and $f_{2}$ are rational maps, then $\bar{\Gamma} \subset \hat{\mathbb{C}}^{2}$ is an irreducible algebraic set.

Proof. First, observe that $\Gamma=\left\{\left(\phi_{1}(z), \phi_{2}(z)\right) ; z \in X\right\}$. Since $\phi_{1}$ and $\phi_{2}$ are rational maps (including polynomials), we have

$$
\bar{\Gamma}=\left\{\left(\phi_{1}(z), \phi_{2}(z) ; z \in \hat{\mathbb{C}}\right\},\right.
$$

and it is an irreducible algebraic set.

3.3. The case of (transcendental) entire maps. Now consider the case $f_{1}$ : $\mathbb{C} \rightarrow \mathbb{C}$ is a transcendental entire map. By the same argument as in Case III above, $X$ cannot be isomorphic to the Riemann sphere. However, we cannot exclude the case $X \cong \mathbb{C}^{*}$ :

Example. Consider the (complexified) Arnold family $f_{a, b}(x)=x+a+b \sin (2 \pi x)$ defined on $\mathbb{C} / \mathbb{Z}$. It is conjugate to $g_{a, b}: \mathbb{C}^{*} \rightarrow \mathbb{C}^{*}$ defined by

$$
g_{a, b}(z)=\lambda z \exp \left(-\frac{b i}{2}\left(z-\frac{1}{z}\right)\right)
$$


where $\lambda=e^{a}$. When $a=0, f_{0, b}$ is odd; $f_{0, b}(-x)=-f_{0, b}(x)$. Therefore, $g_{0, b}(1 / z)=$ $1 / g_{0, b}(z)$ and $g_{0, b}$ is semiconjugate to an entire map

$$
h_{b}(w)=w \cos \left(b \sqrt{w^{2}-1}\right)-i \sqrt{w^{2}-1} \sin \left(b \sqrt{w^{2}-1}\right) .
$$

Observe that $\cos z$ and $z \sin z$ are entire functions of $z^{2}$.

Even when $X \cong \mathbb{C}^{*}$, we can get a semiconjugacy from an entire map to $g$ by taking a proper lift to the universal covering so that it still has a polynomial-like restriction. Hence we need only consider the case $X=\mathbb{C}$ and $g$ is an entire map.

However, as we stated in the Introduction, we do not know whether $g$ is transcendental or not, nor whether $f_{2}$ can be a polynomial or even a rational map.

We conclude this section by the following proposition and its application to Theorem 1

Proposition 4 (Bergweiler). A transcendental entire map $f$ cannot be semiconjugate to a polynomial $P$ by an entire map $g$.

Corollary 5. Let $f_{1}$ be a polynomial and $f_{2}$ be a (transcendental) entire map. Assume $f_{1}$ and $f_{2}$ satisfy the assumption of Theorem 1. Then the entire map $g$ in the conclusion is a polynomial.

This proposition will be proved by comparing the speed of divergence at infinity. Let us recall some notation and facts before the proof. For an entire map $\varphi$, let us denote for $r>0$,

$$
M(r, \varphi)=\max _{|z|=r}|\varphi(z)|
$$

Then we have

(1) $\log M(r, \varphi)$ is an increasing convex function of $\log r$ (Hadamard three-circle theorem).

(2) $\frac{\log M(r, \varphi)}{\log r}$ tends to infinity if and only if $\varphi$ is transcendental.

(3) Let $f, g$ be entire maps and let $a=f(0)$. There exists a constant $K>0$ such that

$$
M(r, g \circ f) \geq M\left(K M\left(\frac{1}{2} r, f\right), g\right)
$$

for sufficiently large $r>0$. (See, e.g., [Ha.)

Proof. We prove by contradiction. Assume $g \circ f=P \circ g$ for a polynomial $P$, a transcendental entire map $f$ and an entire map $g$. Then $g$ must be transcendental also. Let $d=\operatorname{deg} P$. Then there exists some $c>0$ such that

$$
\log M(r, P \circ g) \leq d \log M(r, g)+c
$$

for sufficiently large $r>0$. By assumption and fact (3) above, we have

$$
\log M\left(r_{1}, g\right) \leq d \log M(r, g)+c,
$$

where $r_{1}=K M\left(\frac{1}{2} r, f\right)$. In particular, $\log M\left(r_{1}, g\right) / \log M(r, g)$ is bounded above.

On the contrary, we also have

$$
\frac{\log M\left(r_{1}, g\right)}{\log M(r, g)}=\left(\frac{\log M\left(r_{1}, g\right)}{\log r_{1}} / \frac{\log M(r, g)}{\log r}\right) \cdot \frac{\log r_{1}}{\log r} .
$$


Let $r_{0}>0$. By (11), there exists some $C>0$ such that for any $r>r_{0}$,

$$
\begin{aligned}
\frac{\log M(r, g)}{\log r} & \leq \frac{\log r_{1}-\log r}{\log r_{1}-\log r_{0}} \frac{\log M\left(r_{0}, g\right)}{\log r}+\frac{1-\frac{\log r_{0}}{\log r}}{1-\frac{\log r_{0}}{\log r_{1}}} \frac{\log M\left(r_{1}, g\right)}{\log r_{1}} \\
& \leq \frac{\log M\left(r_{0}, g\right)}{\log r}+\frac{\log M\left(r_{1}, g\right)}{\log r_{1}} ;
\end{aligned}
$$

hence $\left(\frac{\log M\left(r_{1}, g\right)}{\log r_{1}} / \frac{\log M(r, g)}{\log r}\right)$ is bounded from below for sufficiently large $r$ by (2). Since $\frac{\log r_{1}}{\log r}$ tends to infinity by (2), it follows that $\frac{\log M\left(r_{1}, g\right)}{\log M(r, g)}$ tends to infinity, so this is a contradiction.

3.4. Minimality of $g$. We conclude this section by showing the minimality of $g$ :

Proposition 6. The $g$ constructed above is minimal. More precisely, if rational or entire maps $\hat{g}$ and $\hat{\phi}_{i}$ satisfy $\hat{\phi}_{i} \circ \hat{g}=f_{i} \circ \hat{\phi}_{i}$ for $i=1,2$ and there is a well-defined branch of $\hat{\phi}_{1}^{-1}$ on $U_{1}$ such that $\hat{\phi}_{2} \circ \hat{\phi}_{1}^{-1}$ is equal to the original analytic conjugacy $\phi$, then there exists a rational or entire map $\psi$ such that $\psi \circ \hat{g}=g \circ \psi$ and $\hat{\phi}_{i}=\phi_{i} \circ \psi$.

Proof. First assume that $X$ in the proof of Theorem 1 is not isomorphic to $\mathbb{C}^{*}$. Let $\hat{X}=\hat{\mathbb{C}}$ or $\mathbb{C}$ according to whether $\hat{g}$ is rational or entire (a polynomial or a transcendental entire map). Roughly speaking (or when $\hat{g}$ is not transcendental), a map

$$
\begin{array}{rlc}
\tilde{\psi}: \quad \hat{X} & \rightarrow & \hat{\mathbb{C}} \times \hat{\mathbb{C}} \\
z & \mapsto & \left(\hat{\phi}_{1}(z), \hat{\phi}_{2}(z)\right)
\end{array}
$$

gives a map from $\hat{X} \rightarrow \Gamma$ (or $\bar{\Gamma}$ ), so we can get a semiconjugacy $\hat{\phi}=\pi^{-1} \circ \tilde{\psi}$ via the normalization $\pi: X \rightarrow \Gamma$.

More precisely, by assumption, there exists a topological disk $\hat{U} \subset \hat{X}$ such that $\hat{\phi}_{1}: \hat{U} \rightarrow U_{1}$ is an isomorphism and $\hat{\phi}_{i} \circ \hat{g}=f_{i} \circ \hat{\phi}_{i}$ on $\hat{U}^{\prime}=\hat{g}^{-1}(\hat{U}) \cap \hat{U}$ for $i=1,2$. Then $\tilde{\psi}(\hat{U})$ is equal to the graph of $\phi$. Therefore $\psi_{0}=\pi^{-1} \circ \tilde{\psi}: \hat{U} \rightarrow U=X_{0}$ is a conjugacy between $\hat{g}$ and $g$. Similarly, we can define a holomorphic semiconjugacy $\psi_{n}=\pi_{n}^{-1} \circ \tilde{\psi}: \hat{g}^{n}(\hat{U}) \rightarrow g^{n}(U)=X_{n}$ (where $\pi_{n}$ and $X_{n}$ are in the proof of Theorem 2) and by taking the direct limit, we obtain a holomorphic semiconjugacy $\psi=\lim \longrightarrow \psi_{n}: \hat{X} \rightarrow X$ with $\pi \circ \psi=\tilde{\psi}$. It is easy to check that $\psi$ has the desired property.

Even when $X \cong \mathbb{C}^{*}$, the same construction works onto $\mathbb{C}^{*}$ and by taking the lift to the universal cover which analytically conjugates the corresponding polynomiallike restrictions, we can get the same conclusion.

\section{The CASE OF DEgREe OnE POLYNOMIAL-LIKE MAPPINGS}

Here we consider the case when $f_{i}: U_{i}^{\prime} \rightarrow U_{i}(i=1,2)$ are polynomial-like mappings of degree one. By the Schwarz lemma, there exists a repelling fixed point $x_{i} \in U_{i}^{\prime}$ for $f_{i}$ and the forward orbit of any $z \in U_{i}^{\prime}$ except $x_{i}$ escapes from $U_{i}^{\prime}$. Now if $f_{1}: U_{1}^{\prime} \rightarrow U_{1}$ and $f_{2}: U_{2}^{\prime} \rightarrow U_{2}$ are analytically conjugate, then $f_{1}^{\prime}\left(x_{1}\right)=f_{2}^{\prime}\left(x_{2}\right)$. On the contrary, if $\lambda=f_{1}^{\prime}\left(x_{1}\right)=f_{2}^{\prime}\left(x_{2}\right)$, then let $\psi_{i}$ be the inverse of a linearizing coordinate, i.e., a holomorphic map defined near the origin such that $\psi_{i}(0)=x_{i}$, $\psi_{i}^{\prime}(0) \neq 0$ and $\psi_{i}(\lambda x)=f_{i}\left(\psi_{i}(x)\right)$. Then $\psi_{2} \circ \psi_{1}^{-1}$ gives an analytic conjugacy between the polynomial-like restrictions. Furthermore, it is well known that we can extend the domain of definition of $\psi_{i}$ to the whole complex plane. This implies 
that we can always take $X=\mathbb{C}, g(z)=\lambda z$ and $\phi_{i}=\psi_{i}$ in the conclusion of Theorem 1. Namely, there exists a linear map $z \mapsto \lambda z$ which is semiconjugate to both $f_{1}$ and $f_{2}$.

However, we may still apply our construction of $g: X \rightarrow X$ which is semiconjugate to both $f_{1}$ and $f_{2}$ in this case to get the minimal $g$. Since we still have expanding dynamics, $X$ is still parabolic or elliptic. We can have all the cases above because if two polynomial-like maps of degree greater than one are analytically conjugate and have repelling fixed points, then we get analytically conjugate polynomial-like maps of degree one by restricting them to some neighborhoods of the fixed points.

However, we have some other cases because we cannot exclude the case when $\operatorname{deg} g=1$, nor the case when there do not exist any polynomial-like restrictions (linear maps on tori, Lattès maps, $g$ or $f_{i}$ are equal to $z^{ \pm d}: \mathbb{C}^{*} \rightarrow \mathbb{C}^{*}$ ) in the proof. When $\operatorname{deg} g=1$, then $X$ must be isomorphic to $\mathbb{C}$, as we have already seen (i.e., $\psi_{i}=\phi_{i}$ is the inverse of the linearizing coordinate).

When $f_{i}$ is a rational map, we can classify the rest of the cases for a triple $\left(f_{i}, g, \psi_{i}\right)$ as follows:

- If $g: X \rightarrow X$ is a linear map on a torus, then $f_{i}$ is a Lattès map.

- If $g(z)=z^{ \pm d}$ and $X=\mathbb{C}^{*}$, then $f_{i}$ can be either

- a power map $\left(\phi_{i}(z)=z\right)$,

- a Chebyshev map $\phi_{i}(z)=z+1 / z$, or

- an integral Lattès example ( $\phi_{i}$ is transcendental).

- If $\phi_{i}(X)=\mathbb{C}^{*}$, then $f_{i}(z)=z^{ \pm d_{i}}$ and $g$ is either a power map $\left(g=f_{i}\right.$, $\left.X=\mathbb{C}^{*}\right)$ or a linear map $\left.g(z)= \pm d_{i} z, X=\mathbb{C}\right)$.

- If $X=\mathbb{C}$ and $\phi_{i}(X)=\hat{\mathbb{C}}$, then $\phi_{i}$ is transcendental when $g$ is Chebyshev and $f_{i}$ is an integral Lattès example. Note that if $g$ is a power map, then it is not semiconjugate to a Chebyshev map or an integral Lattès example by our construction because $0 \in X=\bigcup_{n \geq 0} V^{\prime}$, which implies that the superattractive fixed point 0 lies in the domain of definition of the polynomial-like restriction $g: V^{\prime} \rightarrow V$.

\section{Appendix A. Semiconjugacies of polynomials}

In this Appendix, we give a classification of semiconjugate polynomials. It is based on the results by Ritt $[\mathrm{R}$ and Engstrom [En on decompositions of polynomials in terms of composition. Let $\mathcal{S}$ be the set of all affine conjugacy classes of triples $(f, g, h)$ of polynomials of degree at least two such that $f \circ h=h \circ g$, where we say that two triples $\left(f_{1}, g_{1}, h_{1}\right)$ and $\left(f_{2}, g_{2}, h_{2}\right)$ are affinely conjugate if there exist affine maps $\sigma_{1}, \sigma_{2}$ such that

$$
\begin{aligned}
& f_{2}=\sigma_{1} \circ f_{1} \circ \sigma_{1}^{-1}, \\
& g_{2}=\sigma_{2} \circ g_{1} \circ \sigma_{2}^{-1}, \\
& h_{2}=\sigma_{1} \circ h_{1} \circ \sigma_{2}^{-1},
\end{aligned}
$$

and we denote $\left(f_{1}, g_{1}, h_{1}\right) \sim\left(f_{2}, g_{2}, h_{2}\right)$. The aim of this Appendix is to classify $[(f, g, h)] \in \mathcal{S}$. 
Theorem 7. Let $[(f, g, h)] \in \mathcal{S}$. If $\operatorname{gcd}(\operatorname{deg} f, \operatorname{deg} h)=d>1$, then there exist polynomials $g_{1}, h_{1}, f_{1}, \hat{h}_{1}, \alpha_{1}$ and $\beta_{1}$ such that

$$
\begin{aligned}
& f \circ h_{1}=h_{1} \circ g_{1}, \quad f_{1} \circ \hat{h}_{1}=\hat{h}_{1} \circ g, \quad h=h_{1} \circ \beta_{1}=\alpha_{1} \circ \hat{h_{1}}, \\
& \operatorname{deg} f_{1}=\operatorname{deg} g_{1}=\operatorname{deg} f, \quad \operatorname{deg} \alpha_{1}=\operatorname{deg} \beta_{1}=d, \quad \operatorname{deg} h_{1}=\operatorname{deg} \hat{h_{1}}=\operatorname{deg} h / d .
\end{aligned}
$$

In particular, if $d<\operatorname{deg} h$, then $\left[\left(f, g_{1}, h_{1}\right)\right],\left[\left(f_{1}, g, \hat{h}_{1}\right)\right] \in \mathcal{S}$.

This theorem implies that we need only consider the case when $\operatorname{deg} f=\operatorname{deg} g$ and $\operatorname{deg} h$ are coprime. More precisely, for a given $\left[\left(f_{0}, g_{0}, h_{0}\right)\right] \in \mathcal{S}$, we can construct a sequence $\left\{\left(f_{0}, g_{n}, h_{n}\right)\right\}_{n=1, \ldots, N}$ and $\left\{\left[\left(f_{n}, g_{0}, \hat{h}_{n}\right)\right]\right\}_{n=1, \ldots, N}$ by applying this theorem repeatedly until we have $\operatorname{gcd}\left(\operatorname{deg} f_{0}, \operatorname{deg} h_{N}\right)=1$ and $\operatorname{gcd}\left(\operatorname{deg} g_{0}, \operatorname{deg} \hat{h}_{N}\right)=1$. Furthermore, if $\operatorname{deg} h_{N}=\operatorname{deg} \hat{h}_{N}>1$, then $\left[\left(f_{0}, g_{N}, h_{N}\right)\right]$ and $\left[\left(f_{N}, g_{0}, \hat{h}_{N}\right)\right]$ lie in $\mathcal{S}$. Otherwise, $f$ and $g_{N}$, and $f_{N}$ and $g$ are affinely conjugate.

Example. Let us consider the example (44) in the Introduction. Namely, let $f_{1}(z)=$ $z\left(c+z^{2}\right)^{3}$ and $f_{2}(z)=z\left(c+z^{3}\right)^{2}$. Since $\operatorname{deg} f_{1}=\operatorname{deg} f_{2}=7$ is a prime number, they are prime polynomials (i.e., $f_{i}$ cannot be decomposed into a composition of polynomials of degree at least two).

Assume there exists a polynomial $h$ such that $f_{1} \circ h=h \circ f_{2}$. If $\operatorname{deg} h$ is divisible by 7 , then by Theorem $7, h$ can be written as

$$
h=f_{1} \circ h_{1}=h_{1} \circ g_{1}
$$

for some $h_{1}$ and $\hat{h}_{1}$. Repeating this argument, we can write $h=f_{1}^{n} \circ h_{n}=\hat{h}_{n} \circ f_{2}^{n}$ for some $n \geq 0, h_{n}$ and $\hat{h}_{n}$ such that $\operatorname{deg} h_{n}$ and $\operatorname{deg} f_{1}=\operatorname{deg} f_{2}$ are coprime. However, Theorem 8 below implies that such an $h_{n}$ exists if and only if $c=0$.

Here we give a complete classification for the case $\operatorname{gcd}(\operatorname{deg} f, \operatorname{deg} h)=1$.

Theorem 8. Assume $[(f, g, h)] \in \mathcal{S}$ satisfies $\operatorname{gcd}(\operatorname{deg} f, \operatorname{deg} h)=1$. Then there exists a representative $\left(f_{0}, g_{0}, h_{0}\right)$ of $[(f, g, h)]$ such that either

- $f(z)=z^{c} P^{b}(z), g(z)=z^{c} P\left(z^{b}\right)$ and $h(z)=z^{b}$, where $c \equiv a \bmod b$, or

- $f=g=T_{a}, h=T_{b}$ are Chebyshev polynomials,

where $a=\operatorname{deg} f(=\operatorname{deg} g)$ and $b=\operatorname{deg} h$.

To prove these theorems, we need the following two facts on decompositions of polynomials with respect to composition. The first one is proved by Ritt $[\mathrm{R}$ :

Theorem 9 (The second Ritt theorem). Let $\varphi_{1}, \varphi_{2}, \psi_{1}$ and $\psi_{2}$ be polynomials of degree at least two such that

$$
F=\varphi_{1} \circ \varphi_{2}=\psi_{1} \circ \psi_{2} .
$$

Assume $a=\operatorname{deg} \varphi_{1}=\operatorname{deg} \psi_{2}$ and $b=\operatorname{deg} \varphi_{2}=\operatorname{deg} \psi_{1}$ are coprime and $a>b$. Then there exist affine maps $\mu, \nu, \sigma_{1}, \sigma_{2}$ such that

$$
\begin{array}{ll}
\hat{\varphi}_{1}=\mu \circ \varphi_{1} \circ \sigma_{1}^{-1}, & \hat{\varphi}_{2}=\sigma_{1} \circ \varphi_{2} \circ \nu, \\
\hat{\psi}_{1}=\mu \circ \psi_{1} \circ \sigma_{2}^{-1}, & \hat{\psi}_{2}=\sigma_{2} \circ \psi_{2} \circ \nu
\end{array}
$$


satisfy either

(1) $\hat{\varphi}_{1}=\hat{\psi}_{2}=T_{a}$ and $\hat{\varphi}_{2}=\hat{\psi}_{1}=T_{b}$, where $T_{n}$ is the Chebyshev polynomial of degree $n$, or

(2) let $0<c<b$ satisfy $a \equiv c \bmod b$. There exists some polynomial $P$ such that $\hat{\varphi}_{2}(z)=\hat{\psi}_{1}(z)=z^{b}$ and

$$
\hat{\varphi}_{1}(z)=z^{c}(P(z))^{b}, \quad \hat{\psi}_{2}(z)=z^{c} P\left(z^{b}\right) .
$$

Remark 10. In the second Ritt theorem, both cases of the conclusion hold only if $b=2$. In particular, if we replace the conclusion (1) by

$\left(1^{\prime}\right) b \geq 3, \hat{\varphi}_{1}=\hat{\psi}_{2}=T_{a}$ and $\hat{\varphi}_{2}=\hat{\psi}_{1}=T_{b}$,

then exactly one of the conclusions (1') or (2) holds.

The second one is proved by Engstrom [En, which is a stronger version of "the first Ritt theorem" $[\mathrm{R}$ :

Theorem 11. Assume $\varphi_{1}, \varphi_{2}, \psi_{1}, \psi_{2}$ are nonconstant polynomials such that

$$
F=\varphi_{1} \circ \varphi_{2}=\psi_{1} \circ \psi_{2} .
$$

Then there exist polynomials $\alpha, \beta, \hat{\varphi}_{1}, \hat{\varphi}_{2}, \hat{\psi}_{1}, \hat{\psi}_{2}$, such that

$$
\begin{array}{lll}
\varphi_{1}=\alpha \circ \hat{\varphi}_{1}, & \psi_{1}=\alpha \circ \hat{\psi}_{1}, & \operatorname{deg} \alpha=\operatorname{gcd}\left(\operatorname{deg} \varphi_{1}, \operatorname{deg} \psi_{1}\right), \\
\varphi_{2}=\hat{\varphi}_{2} \circ \beta, & \psi_{2}=\hat{\psi}_{2} \circ \beta, & \operatorname{deg} \beta=\operatorname{gcd}\left(\operatorname{deg} \varphi_{2}, \operatorname{deg} \psi_{2}\right) .
\end{array}
$$

Indeed, we can say more:

Corollary 12. In the conclusion of Theorem 11, we can also add the condition $\hat{\phi}_{1} \circ \hat{\phi}_{2}=\hat{\psi}_{1} \circ \hat{\psi}_{2}$.

Proof. Assume the conclusion of Theorem [1]. Then we have $\alpha \circ \hat{\phi}_{1} \circ \hat{\phi}_{2} \circ \beta=$ $\alpha \circ \hat{\psi}_{1} \circ \hat{\psi}_{2} \circ \beta$. Since $\beta$ is surjective, we have $\alpha \circ \hat{\phi}_{1} \circ \hat{\phi}_{2}=\alpha \circ \hat{\psi}_{1} \circ \hat{\psi}_{2}$. By applying Theorem 11] again to the decompositions $\alpha \circ\left(\hat{\phi}_{1} \circ \hat{\phi}_{2}\right)=\alpha \circ\left(\hat{\psi}_{1} \circ \hat{\psi}_{2}\right)$, we can see that there exist polynomials $\Phi, \Psi, \beta_{1}$ such that

$$
\hat{\phi}_{1} \circ \hat{\phi}_{2}=\Phi \circ \beta_{1}, \quad \hat{\psi}_{1} \circ \hat{\psi}_{2}=\Psi \circ \beta_{1}
$$

and

$$
\operatorname{deg} \beta_{1}=\operatorname{gcd}\left(\operatorname{deg} \hat{\phi}_{1} \circ \hat{\phi}_{2}, \hat{\psi}_{1} \circ \hat{\psi}_{2}\right)=\operatorname{deg} \hat{\phi}_{1} \circ \hat{\phi}_{2}=\operatorname{deg} \hat{\psi}_{1} \circ \hat{\psi}_{2}
$$

In particular, $\Phi$ and $\Psi$ are affine. Therefore, we have

$$
\hat{\phi}_{1} \circ \hat{\phi}_{2}=\Phi \circ \Psi^{-1} \circ \hat{\psi}_{1} \circ \hat{\psi}_{2} .
$$

It is easy to check that the conclusion holds if we replace $\hat{\psi}_{1}$ by $\Phi \circ \Psi^{-1} \circ \hat{\psi}_{1}$.

Now we prove Theorem 7 First of all, the following lemma easily follows from Corollary 12,

Lemma 13. Let $[(f, g, h)] \in \mathcal{S}$. Then there exist polynomials $\phi_{1}, \phi_{2}, \psi_{1}, \psi_{2}, h_{1}, \hat{h}_{1}$ such that

$$
\begin{aligned}
f & =\phi_{1} \circ \phi_{2}, & g & =\psi_{1} \circ \psi_{2}, \\
h & =\phi_{1} \circ \hat{h}_{1}=h_{1} \circ \psi_{2}, & \phi_{2} \circ h_{1} & =\psi_{1} \circ \hat{h}_{1}, \\
\operatorname{deg} \phi_{1} & =\operatorname{deg} \psi_{2}=\operatorname{gcd}(\operatorname{deg} f, \operatorname{deg} h), & \operatorname{deg} \psi_{1} & =\operatorname{deg} \phi_{2}, \\
\operatorname{deg} h_{1} & =\operatorname{deg} \hat{h}_{1} . & &
\end{aligned}
$$


In particular, the following diagram commutes:

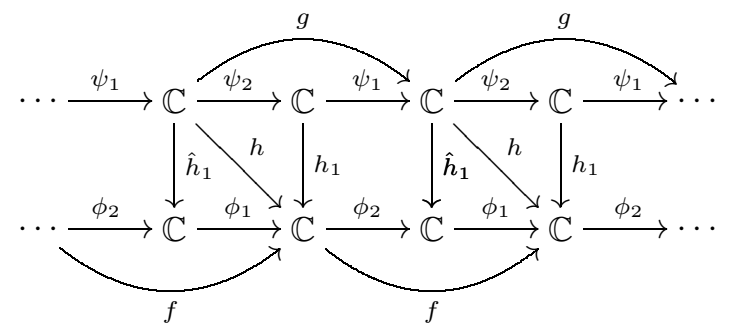

and if $\operatorname{deg} h_{1} \geq 2$, we have $\left[\left(f, g_{1}, h_{1}\right)\right],\left[\left(f_{1}, g, \hat{h}_{1}\right)\right] \in \mathcal{S}$ where $f_{1}=\phi_{2} \circ \phi_{1}$ and $g_{1}=\psi_{2} \circ \psi_{1}$.

Theorem 7 is an immediate consequence of the diagram (3).

Proof of Theorem 8 . Let $[(f, g, h)] \in \mathcal{S}$ with $\operatorname{gcd}(\operatorname{deg} f, \operatorname{deg} h)=1$. By the second Ritt theorem, there exist affine maps $\mu, \nu, \sigma_{1}, \sigma_{2}$ such that

$$
\begin{array}{ll}
\hat{\varphi}_{1}=\mu \circ f \circ \sigma_{1}^{-1}, & \hat{\varphi}_{2}=\sigma_{1} \circ h \circ \nu, \\
\hat{\psi}_{1}=\mu \circ h \circ \sigma_{2}^{-1}, & \hat{\psi}_{2}=\sigma_{2} \circ g \circ \nu
\end{array}
$$

satisfy the conclusion of Theorem 9 (note that we do not know whether $a=\operatorname{deg} f>$ $b=\operatorname{deg} h$ or not). By taking an affine conjugacy, we may further assume that $\mu$ and $\sigma_{2}$ are the identity, so it follows that $h=\hat{\psi}_{1}$.

First assume $a>b$. Then we have

$$
\hat{\varphi}_{2}=\hat{\psi}_{2}=h=\sigma_{1}^{-1} \circ \hat{\varphi}_{2} \circ \nu
$$

are either the power map or the Chebyshev polynomial of degree $b$. If they are Chebyshev, then $\nu$ and $\sigma_{1}$ are also equal to the identity and we are done. If they are power maps, then $\nu(z)=\nu \cdot z$ and $\sigma_{1}(z)=\sigma \cdot z$ are linear maps with $\sigma^{b}=\nu$. Let us denote $\hat{\phi}_{1}(z)=z^{c}(P(z))^{b}$ and $\hat{\psi}_{2}(z)=z^{c} P\left(z^{b}\right)$. Then $f(z)=z^{c}\left(P_{1}(z)\right)^{b}$ and $g(z)=z^{c}\left(P_{1}\left(z^{b}\right)\right)$, where $P_{1}(z)=\sigma^{c} P\left(\sigma^{b} z\right)$, so we are also done.

Now assume $a<b$. If $\hat{\phi}_{i}$ and $\hat{\psi}_{i}$ are Chebyshev, then we have the same conclusion as above. So the remaining case is the following:

(3) Let $0<c<a$ satisfy $b \equiv c \bmod a$. There exists some polynomial $P$ such that $\hat{\varphi}_{1}(z)=\hat{\psi}_{2}(z)=z^{a}$ and

$$
\hat{\varphi}_{2}(z)=z^{c}(P(z))^{a}, \quad \hat{\psi}_{1}(z)=z^{c} P\left(z^{a}\right) .
$$

Even in this case, by considering $\left(f^{\circ n}, g^{\circ n}, h\right)$ for sufficiently large $n$, we have $\operatorname{deg} f^{\circ n}=a^{n}>b$, and $\operatorname{gcd}\left(a^{n}, b\right)=1$. Thus again we can apply the second Ritt theorem and $h$ has the form

$$
h(z)=A(z+B)^{b}+C .
$$

(Observe that $h$ is not Chebyshev because then $f$ and $g$ must be Chebyshev.) Furthermore, $h(z)$ has a symmetry: For an $a$-th root of unity $\omega$, we have $h(\omega z)=$ $\omega^{c} h(z)\left(=\omega^{b} h(z)\right)$. This implies $B=C=0$, i.e., $h(z)=A z^{b}$. Hence $P(z)=$ $A z^{(b-c) / a}$ and $\hat{\phi}_{2}(z)=A z^{b}=h(z)$. Therefore, the triple $(f, g, h)$ is affinely conjugate to $\left(z^{a}, z^{b}, z^{a}\right)$, so it is the first case of the conclusion of Theorem 8 . 
A generalization of Ritt's theorem for rational maps is an open problem. Hence we cannot classify semiconjugacies of rational maps. A generalization to Laurent polynomials was recently done by Zieve [Z].

\section{ACKNOWLEDGMENT}

The author thanks Xavier Buff, Shaun Bullett, Arnaud Chéritat, Shu Kawaguchi and Tomoki Kawahira for valuable discussions. He also thanks Adam Epstein and Alexandre Eremenko for helpful comments, and he thanks Walter Bergweiler for explaining that a transcendental entire map cannot be globally semiconjugate to a polynomial.

\section{REFERENCES}

[BE] X. Buff, A. L. Epstein. From local to global analytic conjugacies. Ergodic Theory Dynam. System 27 (2007), 1073-1094. MR.2342966 (2008g:37039)

[BS] S. Bullett, C. Penrose. Regular and limit sets for holomorphic correspondences. Fund. Math. 167 (2001), 111-171. MR1816043 (2002d:37068)

[C] E. M. Chirka. Complex analytic sets. Translated from the Russian by R. A. M. Hoksbergen. Mathematics and its Applications (Soviet Series), 46. Kluwer Academic Publishers Group, Dordrecht, 1989. MR 1111477 (92b:32016)

[DH] A. Douady, J. H. Hubbard. On the dynamics of polynomial-like mappings. Ann. Sci. École Norm. Sup. (4) 18 (1985), 287-343. MR816367 (87f:58083)

[En] H. T. Engstrom. Polynomial substitutions. Amer. J. Math. 63 (1941), 249-255. MR0003599 $(2: 242 \mathrm{f})$

[Er] A. Eremenko. Some functional equations connected with the iteration of rational functions. Algebra i Analiz 1 (1989), 102-116; translation in Leningrad Math. J. 1 (1990), 905-919. MR:1027462 (90m:30030)

[Ha] W. K. Hayman. Meromorphic functions. Oxford Mathematical Monographs, The Clarendon Press, Oxford, 1964. MR0164038 (29:1337)

[I] H. Inou. Combinatorics and topology of straightening maps II: discontinuity. In preparation.

[M] J. Milnor. On Lattès maps. Dynamics on the Riemann Sphere. Eds. P. Hjorth and C. L. Petersen. A Bodil Branner Festschrift, European Mathematical Society, 2006, pp. 9-43. MR2348951 (2008e:37001)

[R] J. F. Ritt. Prime and composite polynomials. Trans. Amer. Math. Soc. 23 (1922), 51-66. MR.1501189

[Z] M. E. Zieve. Decompositions of Laurent polynomials. Preprint.

Department of Mathematics, Kyoto University, Kyoto 606-8502, Japan 